\title{
Fuzzy Axiomatic Approach to Blue-green Infrastructure Strategy Selection: A Sustainability Perspective
}

\author{
Desmond Eseoghene Ighravwe $\left({ }^{\square}\right)$ \\ Department of Electrical and Electronic Engineering Science, University of Johannesburg, South Africa \\ ighravwedesmond@gmail.com \\ Daniel Mashao \\ Department of Electrical and Electronic Engineering Science, University of Johannesburg, South Africa
}

\begin{abstract}
Flood risk management (FRM) is a global problem that has received significant attention from scholars. Their interest is on the minimisation of flood occurrence and its aftermath. This study uses FRM sustainability criteria to contribute a framework to the interest mentioned above. It designed the framework for a blue-green technology selection using a best-worst method, fuzzy axiomatic method and VIKOR method. Data from Lekki, Lagos, Nigeria, was used to test this framework applicability. Six blue-green technologies, which include bypass floodway, rainwater and floodwater harvesting, and porous pavement, were considered as potential technology for the case study. From the framework implementation results, this study observed that techno-economic criteria contributed about $88.18 \%$ to the ranking of blue-green technology. The framework identified rainwater and floodwater harvesting as the most suitable blue-green technologies for a community. It also identified a bypass floodway as the least suitable blue-green technologies for a community. With these results, the proposed framework will aid decisionmakers strategic and tactical criteria that can be used to evaluate blue-green technology selection. Keywords - Flood, multi-criteria, blue-green technology, sustainability, fuzzy logic.
\end{abstract}

\section{Introduction}

As the earth experiences climate change, stakeholders have organised several fora on how to handle the effect of this change effectively. Flood and drought are among the aftermath of this change. Scholars have reported that flood destroys several communities annually, especially in developing countries with urban plans [1], [2]. One of the recommendations of scholars is the use of a sustainability approach to arrest this problem [3], [4]. The issue of flood management should not be considered as an engineering problem [4]. It requires a multi-disciplinary approach to harness the contributions from other disciplines during flooding management. For instance, the decision on appropriate blue-green technology for flood-prone areas can be solved from a multi-disciplinary perspective.

This perspective is desired because of the need to find a sustainable solution to flooding. It is, therefore, the responsibility of stakeholders to include sustainability into consideration when such a solution is recommended. Sustainability - which is the analysis of technical, social, economic and environmental requirements of a system - helps to cater to the present and future needs of different generations. The criteria that constitute sustainability requirements are system dependent. Hence, decision-makers are required to carry out a preliminary analysis of these requirements and present them in a framework to stakeholders - investors, government, and public. Since some of the criteria in a sustainable framework can only be expressed in linguistic forms, it is the duty of decision-makers to make their findings as simple as possible to the stakeholders. To create a simplified framework that embeds sustainability requirements, scholars have accepted multi-criteria modelling approaches as being robust for this purpose.

Multi-criteria decision-making (MCDM) tools combine a system's requirements to determine the best course of action. Examples of MCDM tools are VIKOR, TOPSIS, and axiomatic method, just to mention a few. Their applications involve careful planning of system requirements to generate practical solutions for real-world problems. Currently, research domains such as energy, maintenance, and supply chain management have established fundamental elements that constitute system requirements for the design of MCDM frameworks for domains mentioned above [5]. Unfortunately, scholars in flood risk management (FRM) domain are yet proposed a framework that contains sustainability 
requirements. This study aims to use stakeholders' perspectives requirements to develop a sustainability framework for FRM. In the framework, the socio-economic requirements of a community were considered [2]. Apart from these needs, this study considered the technical, environmental, and policy requirements for blue-green technology adoption. Three MCDM tools were used to combine the requirements mentioned above synergically. The framework determines sub-criteria importance using a best-worst method. A fuzzy axiomatic method was used to relate design requirements with system requirements for alternative solutions to blue-green technology adoption. VIKOR was used to combine the sustainability criteria to determine the most suitable blue-green technology for a system.

\section{Flood Risk Management}

FRM is an activity that deals with flood preparation, prevention and mitigation, just to mention a few [6]. Because of the importance of FRM to human survival, several studies have been published on FRM[1], [7]-[9]. For example, [9] stated that the justification for selecting a flood risk strategy could be improved when information on flood-risk design is combined with a decision-making model for FRM. [10] presented a methodology for household vulnerability assessment to flood. Their work considered the damages caused by flood to property, water contamination, physical and mental impairments, and exposure to hazard. [7] noted that while pushing for a flood strategy, there is a need to incorporate the issue of landscape quality to protect cultural heritage, and to account for the spatial and building requirements of a community.

To deploy an FRM strategy, the contributions of stakeholders are required. When their contributions are considered, it will not only address the immediate need for flood control; it will also sustain the environment for the next generation [11]. To improve the FRM decision-making process, [12] presented a framework that aggregates social requirements, such as household vulnerability, to flood management. Beyond such requirements, it is equally essential that a decision support system for FRM contains economic, technical, and environmental requirements. This consideration is suitable for longterm analysis of flood control [13]. Another critical issue in the design of an FRM strategy is uncertainty consideration.

The uncertainty in an FRM strategy can be understood by classifying a model as a preventive or mitigation model [14]. This classification improves how an FRM model adjusts to the emerging needs of communities [2]. Scholars have used different approaches to solve this problem. For instance, [15] used a hydraulic modelling approach to evaluate policies for FRM. They generated flood risk maps using used a regular levee system. The generated maps showed that the non-structural method reduces the damages caused by a flood. Apart from policy uncertainty, financing an FRM strategy introduces uncertainty into flood management [16]. It is, therefore, imperative that government policies and the socio-economic needs of a community are understood before settling for any FRM strategy. This issue can be addressed by using stakeholders inputs to design an FRM programme [2], [17].

Since stakeholders' inputs increase the dynamics of FRM, scholars need to understand a community's evolving social and physical needs. Hence, attention must be given to the relationship between policymakers' expectations and human behaviours [8]. This is because robust flood management depends on a community's socio-economic and environmental needs, as well as its flood policy [17]. There are several aspects of this relationship that should be considered as scholars seek a robust FRM strategy for a community. First, governments should continuously evaluate the effectiveness of water channels and storage systems, and flood warning and evacuation systems. The evaluation process should cover the techno-economic considerations of these systems [18]. Second, insurance policy needs to be provided for people living in flood-prone areas [17]. Third, more emphasis should be given to the non-structural measures of FRM over the structural measures [2].

From the forthgoing, three facts emerged from the literature. FRM problem is a multi-criteria problem, and analysis on this problem must include the socio-economic benefits for adopting a strategy. Stakeholders' inputs are essential to the success of an FRM strategy. Uncertainty must be considered when selecting an FRM strategy for a community. Motivated by these findings, the current study presents a framework that used the facts as mentioned above to design a decision-making tool for bluegreen technology selection for developing countries. 


\section{Methodology}

This study considered the FRM problem as a decision-making problem because there is often a conflict of interest among stakeholders that constitute a team for an FRM strategy adoption [19]. Hence, it uses multi-criteria tools to address the blue-green technology problem. Figure 1 illustrates the relationships between the different MCDM tools in this work. Because of the difficulty of using a quantitative approach to select flood management strategy, this study considers qualitative information for the current problem analysis.

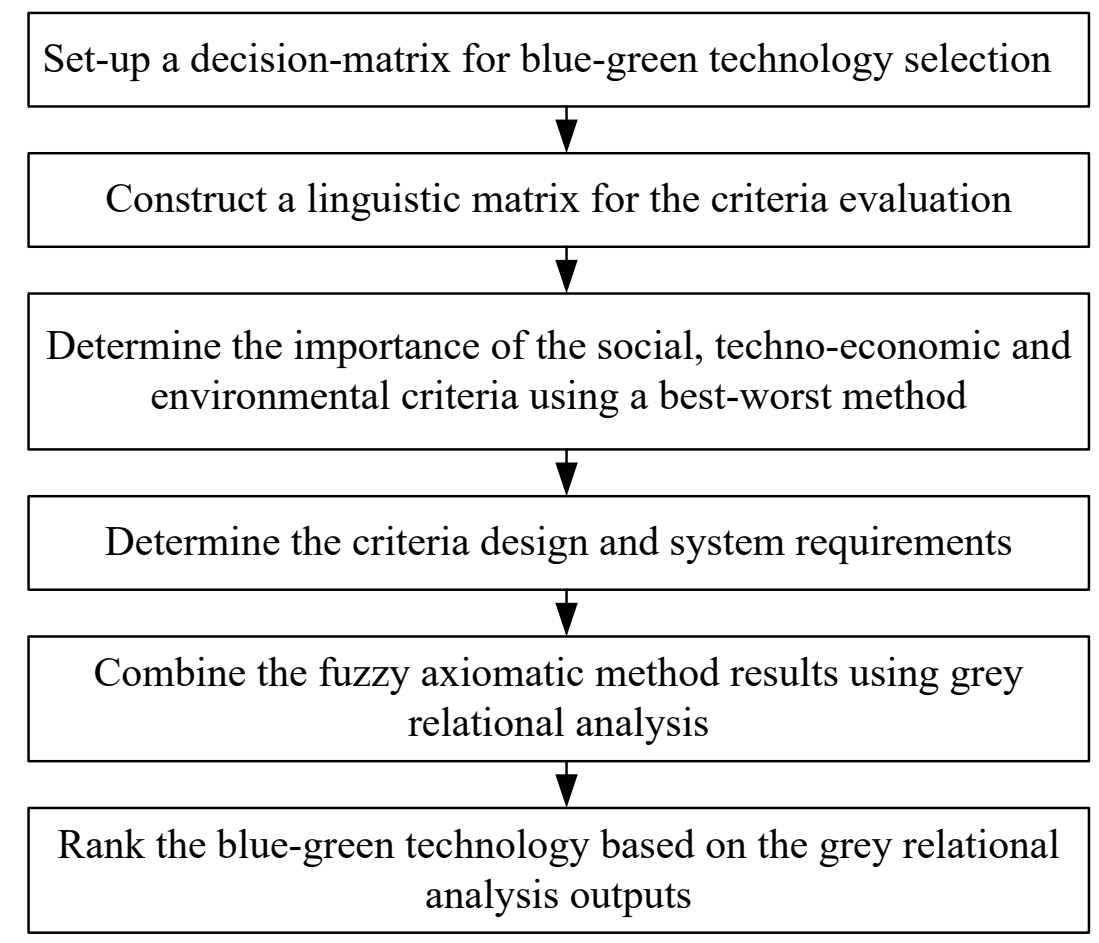

Fig. 1. Schematic flow of the proposed methodology

\subsection{Best worst method}

This study addresses the problem of optimal weight determination for criteria using a best-worst method. The method uses sets of non-linear equations to determine criteria importance. It synchronises experts' judgments through non-linear equations. The equations are organised in a way that the distance between the best and worst criteria are optimised for all sub-criteria [20]; this makes the best-worst method a unique method for criteria importance evaluation. Furthermore, this attribute gives it an edge over an analytical hierarchy process [21]. The steps for implementing a grey best worst method are expressed as follows [22]:

Step 1: Select the criteria for the blue-green technology evaluation.

Step 2: Determine a scale for the socio-economic, technical, and environmental criteria evaluation.

Step 3: Constitute a panel of experts that will evaluate the criteria importance.

Step 4: Determine the best (Equation 1) and worst (Equation 2) criteria.

Error! Bookmark not defined. $A_{B}=\left\{a_{B 1}, a_{B 2}, a_{B 3}, \ldots, a_{B n}\right\}$

$$
A_{W}=\left\{a_{1 W}, a_{2 W}, a_{3 W}, \ldots, a_{n W}\right\}
$$


where $A_{B}$ and $A_{W}$ denote the vector of the best and worst criteria for a decision-making process, respectively.

Step 5: Construct a pairwise comparison of the criteria, starting from the best criterion to other criteria in decreasing order.

Step 6: Use Equations (3) to (7) to determine the criteria importance.

\section{Min $\xi$}

s.t.

$$
\begin{aligned}
& \left|\frac{w_{B}}{w_{i}}-a_{B i}\right| \leq \xi \\
& \left|\frac{w_{i}}{w_{B}}-a_{w i}\right| \leq \xi \\
& \sum_{i=1}^{n} w_{i}=1 \\
& 0 \leq w_{i} \leq 1 \quad i=1,2,3, \ldots n
\end{aligned}
$$

Where $w_{i}$ denotes criterion $i$ weight.

\subsection{Fuzzy axiomatic method}

Axiomatic method is a multi-criteria approach that incorporates design requirements into a decisionmaking process. It uses information content to rank alternatives based on the relationship between design and system requirements. Because of the uniqueness of this method, scholars have used fuzzy logic to increase its application [23]. Its fuzzy version uses fuzzy numbers to convert linguistic terms to crisp values using either triangular or trapezoidal fuzzy numbers. The steps below give a summarised description of a weighted fuzzy axiomatic method:

Step 1: Identify alternative solutions to a decision-making process.

Step 2: Select the criteria for the evaluation process and state linguistic terms for the criteria. Also, select an appropriate method for the criteria importance evaluation.

Step 3: Identify experts that will evaluate the identified alternatives in step 1.

Step 4: Define the criteria's design requirements using the use of the experts' judgements.

Step 5: Aggregate the experts' judgement using an appropriate method. The current study used [24] aggregation expressions in Equations (8) to (11) to aggregate experts' judgements and [23] weighted expression for trapezoidal fuzzy numbers to generate the weighted aggregated values for functional requirements (Equation 12).

$$
\begin{aligned}
& l_{i}=\min \left\{\left(l_{i k}\right)\right\} \\
& m_{i}^{2}=\frac{1}{k} \sum_{k \in K} m_{i k}^{1}
\end{aligned}
$$




$$
\begin{aligned}
& m_{i}^{2}=\frac{1}{k} \sum_{k \in K} m_{i k}^{2} \\
& u_{i}=\max \left\{\left(u_{i k}\right)\right\}
\end{aligned}
$$

$$
f r_{i}=\left(\left(l_{i}+\left(m_{1 i}-l_{i}\right) \times w_{i}\right), m_{1 i}, m_{2 i},\left(u_{i}+\left(u_{i}-m_{2 i}\right) \times w_{i}\right)\right)
$$

Step 6: Evaluate the alternatives common areas using Equation (13). This area is the triangle created by the intersection between a system and design requirements (Figure 2).

$$
C R=\frac{\left(\delta_{2}-\alpha_{1}\right)^{2}}{2\left(\delta_{2}-\beta_{2}+\beta_{1}-\alpha_{1}\right)}
$$

Step 7: Compute the alternatives' system requirements using Equation (14).

$$
S R=\frac{\tau_{1} \delta_{1}+1 / 3\left(\tau_{1}+\delta_{1}\right)^{2}+\beta_{1} \alpha_{1}+1 / 3\left(\beta_{1}+\alpha_{1}\right)^{2}}{\tau_{1}+\delta_{1}-\beta_{1}-\alpha_{1}}
$$

Step 8: Calculate the alternatives' chance of meeting the design requirements using Equation (15)

$$
p_{i j}=\frac{C R}{S R}
$$

Step 9: Compute the criteria information contents using Equation (16) and the alternatives' total information content using Equation (17).

$$
\begin{aligned}
& I_{i j}=\log _{2}\left(\frac{1}{p_{i j}}\right) \\
& A_{i}=\sum_{j=1}^{n} I_{i j}
\end{aligned}
$$

Step 10: Rank the alternative based on the lowest total information content, the better the alternative. 


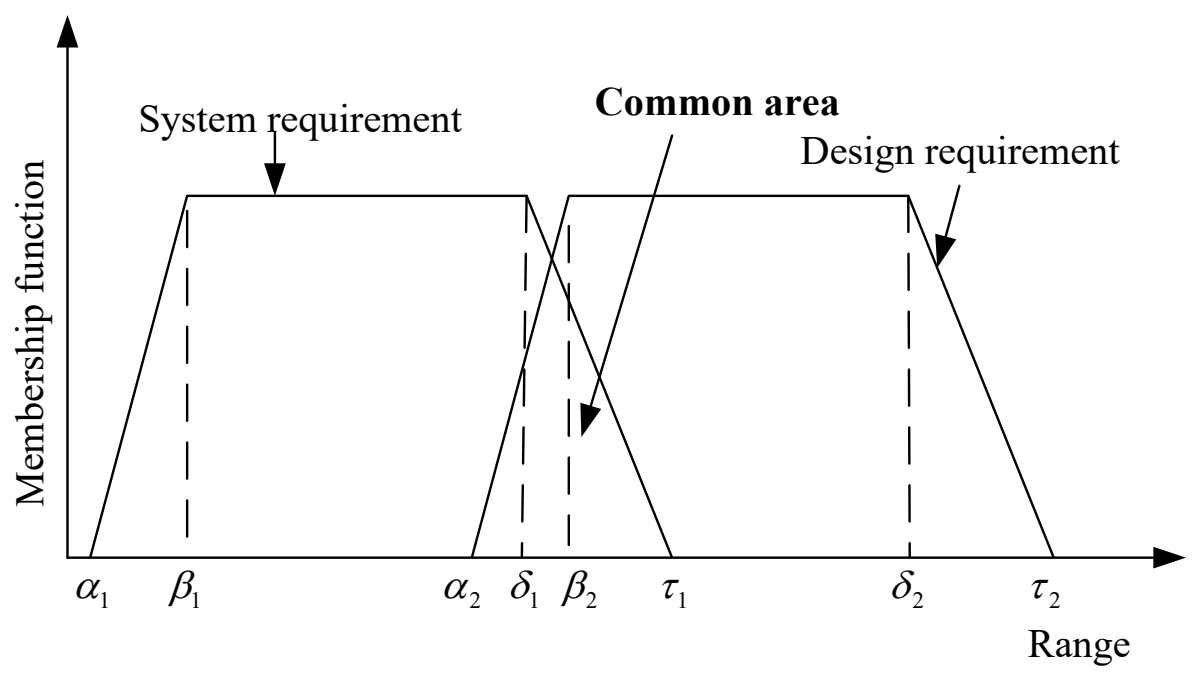

Fig. 2. Common area between a design and a system requirement

\subsection{Grey relational analysis}

The steps below outlines the procedure for this method application [25], [26]:

Step 1: Construct a decision-matrix for a MCDM problem (Equation 18)

$D=\left[\begin{array}{ccc}r_{11} & r_{12}, \ldots, & r_{1 m} \\ r_{21} & r_{22}, \ldots, & r_{2 m} \\ r_{n 1} & r_{n 2}, \ldots, & r_{n m}\end{array}\right]$

Step 2: Normalise the information in the matrix. Criteria which are benefit-oriented are normalised with Equation (19), while Equation (20) is used to normalised criteria which are cost-oriented.

$x_{i}(k)=\frac{r_{i}(k)-r_{i}^{\min }}{r_{i}^{\max }+r_{i}^{\min }}$

$x_{i}(k)=\frac{r_{i}^{\max }-r_{i}(k)}{r_{i}^{\max }+r_{i}^{\min }}$

where $x_{i}(k)$ and $r_{i}(k)$ denote the normalised and real values for criteria $\mathrm{k}$ for alternative $\mathrm{i}, r_{i}^{\max }$ and $r_{i}^{\max }$ denote the maximum and minimum values of criterion $\mathrm{k}$.

Step 3: Specific an ideal sequence $\left(x_{0}(k)\right)$ for the evaluation process.

Step 4: Evaluate the deviation of the normalised decision matrix from the specified ideal sequence. The process requires that an identification coefficient value be defined for the evaluation process. 


$$
\xi_{i}(k)=\frac{\Delta_{\min }+\xi \Delta_{\max }}{\Delta_{o, i}(k)+\xi \Delta_{\max }}
$$

$$
\Delta_{o, i}(k)=\left\|x_{o}(k)-x_{i}(k)\right\|
$$

$$
\Delta_{\text {min }}=\min _{\forall i} \min _{\forall k}\left\|x_{o}(k)-x_{i}(k)\right\|
$$

$$
\Delta_{\max }=\max _{\forall i} \max _{\forall k}\left\|x_{o}(k)-x_{i}(k)\right\|
$$

Step 5: Determine the alternatives' grey relational grade by combining the grey relational coefficients with criteria importance (Equation 25).

$$
\gamma(k)=\frac{1}{n} \sum_{i=1}^{n} w_{i} \xi_{i}(k)
$$

Step 6: Rank the alternatives using a higher-the-better approach.

\section{Case Study}

Lekki, a community in Lagos, Nigeria, was used as a case study. This community, which was designed as a blue-green city, has about 3.4 and 1.9 million for residential and non-residential people, respectively. Its large population is because it is a free trade zone. Dangote oil refinery is among the multi-billion-dollar project in this community. Its annual temperature is about $3.3^{\circ} \mathrm{C}$ annual, while its maximum monthly rainfall is $91.6 \mathrm{~mm}$. This location's geographical coordinates are $6^{\circ} 25^{\prime} 0^{\prime \prime}$ North and $4^{\circ} 6^{\prime} 0$ " East (Figure 3). This community has been transformed from a rural community to an urban community. However, the poor implementation of its urban development plan has made it to become a flood-prone community. This problem is affecting its commercial activities, such as fishing and tourism.

During the implementation of the proposed framework (Figure 1), this study considered six blue-green technologies (Table 1). These technologies were evaluated based on the criteria in Table 2. This study used a well-structured questionnaire to obtain information for the evaluation process [27], [28]. The questionnaire has two sections. Information about the criteria importance is contained in the first section; blue-green technologies information is contained in the second section. Three of the decisionmakers, i.e., experts, were selected from academics and industry. These experts' minimum working experience and qualifications are eight years and a masters' degree in civil and environmental engineering. Their contributions to the evaluation process are 0.2 for Expert 1 (E1), 0.3 for Expert 2 (E2), and 0.5 for Expert 3 (E3) [29]. The experts were asked to evaluate the criteria importance based on the linguistic variables in Table 3 . 


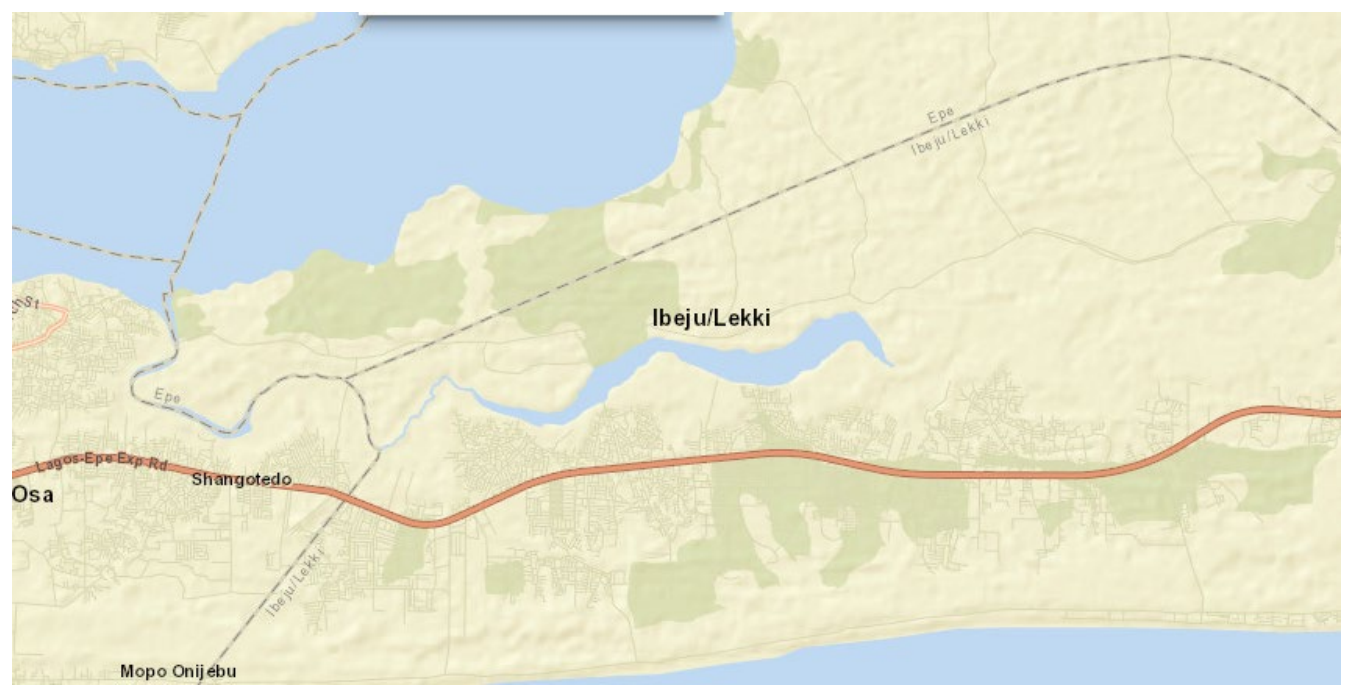

Fig. 3. Lekki spatial location [30]

Table 1. Selected blue-green technology

\begin{tabular}{|l|l|}
\hline Blue-green Technology & Description \\
\hline $\begin{array}{l}\text { Rainwater and } \\
\text { floodwater harvesting } \\
\left(\mathrm{A}_{1}\right)\end{array}$ & This technology harvests runoff waters for agriculture purposes. \\
\hline $\begin{array}{l}\text { Pumped hydropower } \\
\text { systems }\left(\mathrm{A}_{2}\right)\end{array}$ & $\begin{array}{l}\text { This technology uses flood water stored in a reservoir to generate } \\
\text { hydropower. }\end{array}$ \\
\hline Transport basin $\left(\mathrm{A}_{3}\right)$ & $\begin{array}{l}\text { This technology is used to manage stormwater runoff. It has the capacity to } \\
\text { prevent improve water quality in an area. }\end{array}$ \\
\hline Porous pavement $\left(\mathrm{A}_{4}\right)$ & This technology uses a porous pavement to manage stormwater. \\
\hline Retention lake $\left(\mathrm{A}_{5}\right)$ & $\begin{array}{l}\text { This technology uses an artificial pond to prevent downstream erosion and } \\
\text { flooding. }\end{array}$ \\
\hline Bypass floodway $\left(\mathrm{A}_{6}\right)$ & $\begin{array}{l}\text { This technology uses a sizeable man-made channel to control excess flood } \\
\text { waters. }\end{array}$ \\
\hline
\end{tabular}

Table 2. Evaluation criteria

\begin{tabular}{|c|c|}
\hline Criteria & Description \\
\hline \multicolumn{2}{|l|}{ Economic criteria } \\
\hline Investment cost $\left(\mathrm{C}_{11}\right)$ & $\begin{array}{l}\text { This criterion measures the cost that will be incurred in order to } \\
\text { purchase a blue-green technology for a community [31]. }\end{array}$ \\
\hline $\begin{array}{l}\text { Operation and maintenance } \\
\text { cost }\left(\mathrm{C}_{12}\right)\end{array}$ & $\begin{array}{l}\text { This criterion measures the running expenses that will be incurred in } \\
\text { order to provide acceptance flood management service to a } \\
\text { community [31]. }\end{array}$ \\
\hline $\begin{array}{l}\text { Ease of partnership funding } \\
\left(\mathrm{C}_{13}\right)\end{array}$ & $\begin{array}{l}\text { This criterion measures the ease of getting a public-private } \\
\text { partnership that will be used to execute a blue-green technology in a } \\
\text { community (Experts). }\end{array}$ \\
\hline Implementation cost $\left(\mathrm{C}_{14}\right)$ & $\begin{array}{l}\text { This criterion measures the cost of implementing a selected blue- } \\
\text { green technology in a community [7]. }\end{array}$ \\
\hline \multicolumn{2}{|l|}{ Environmental } \\
\hline $\begin{array}{lll}\begin{array}{l}\text { Adaptability } \\
\text { change }\left(\mathrm{C}_{21}\right)\end{array} & \text { to } & \text { climate } \\
\end{array}$ & $\begin{array}{l}\text { This criterion measures the ease of incorporating a blue-green } \\
\text { technology into the climate change policy of a community [4]. }\end{array}$ \\
\hline Land use $\left(\mathrm{C}_{22}\right)$ & $\begin{array}{l}\text { This criterion evaluates looks that the impact of a blue-green strategy } \\
\text { at it affects the use of land for other productive use (Yazdandoost and } \\
\text { Bozorgy, 2008) }\end{array}$ \\
\hline Air quality improvement $\left(\mathrm{C}_{23}\right)$ & $\begin{array}{l}\text { This criterion looks at the impact of a blue-green technology } \\
\text { concerning its implications of air quality in an area (Experts). }\end{array}$ \\
\hline Habitat connectivity $\left(\mathrm{C}_{24}\right)$ & $\begin{array}{l}\text { This criterion measures the impact of a blue-green technology as it } \\
\text { affects the relationship among different habitats in a community } \\
\text { (Experts). }\end{array}$ \\
\hline
\end{tabular}




\begin{tabular}{|l|l|}
\hline Social & \\
\hline $\begin{array}{l}\text { Improvement in water quality } \\
\left(\mathrm{C}_{31}\right)\end{array}$ & $\begin{array}{l}\text { This criterion evaluates the impact of a blue-green technology on the } \\
\text { provision of quality water for a community (Experts). }\end{array}$ \\
\hline $\begin{array}{l}\text { Public realm improvement } \\
\left(\mathrm{C}_{32}\right)\end{array}$ & $\begin{array}{l}\text { This criterion measures how a blue-green technology will affect areas } \\
\text { that are allocated for public use [11]. }\end{array}$ \\
\hline Job generation $\left(\mathrm{C}_{33}\right)$ & $\begin{array}{l}\text { This criterion evaluates the opportunity opportunities that will be } \\
\text { created based on a selected blue-green technology for a community } \\
\text { (Experts). }\end{array}$ \\
\hline $\begin{array}{l}\text { Technology acceptability by } \\
\text { the public }\left(\mathrm{C}_{34}\right)\end{array}$ & $\begin{array}{l}\text { This criterion measures the level of acceptance that a blue-green } \\
\text { technology will receive from the members of a community (Experts). }\end{array}$ \\
\hline Technical $\left(\mathrm{C}_{4}\right)$ & $\begin{array}{l}\text { This criterion evaluates the ease at which a blue-green technology can } \\
\text { be incorporated into an urban community plan (Experts). }\end{array}$ \\
\hline urban plan $\left(\mathrm{C}_{41}\right)$ & $\begin{array}{l}\text { It denotes the capacity of a blue-green technology to prevent floods } \\
\text { [31] [31]. }\end{array}$ \\
\hline Resilience $\left(\mathrm{C}_{43}\right)$ & $\begin{array}{l}\text { It denotes the capacity of the blue-green technology system to recover } \\
\text { from floods [31]. }\end{array}$ \\
\hline $\begin{array}{l}\text { Technological capacity and } \\
\text { support }\left(\mathrm{C}_{44}\right)\end{array}$ & $\begin{array}{l}\text { This criterion looks at the level of technical skills that are available } \\
\text { for the implementation of a blue-green technology in a community } \\
\text { (experts). }\end{array}$ \\
\hline Sensitivity $\left(\mathrm{C}_{45}\right)$ & $\begin{array}{l}\text { The ability of technology to adapt to change in the operating } \\
\text { condition of an environment; this criterion overs the robustness and } \\
\text { flexibility of a technology [31]. }\end{array}$ \\
\hline Safety $\left(\mathrm{C}_{46}\right)$ & $\begin{array}{l}\text { This criterion measures how safe blue-green technology is when } \\
\text { installed in a community [9]. }\end{array}$ \\
\hline
\end{tabular}

The selected experts evaluated the criteria importance using the linguistic terms in Table 3 - their responses are presented in Table 4. This study developed four optimisation models. The first model was developed for the economic criteria evaluation, the second model was developed for the environmental criteria evaluation, the third model was developed for the social criteria evaluation, and the last model was developed for the technical criteria evaluation. The formulated models were solved using Microsoft excel solver (Table 5).

Table 3. Linguistic terms and their crisp values

\begin{tabular}{lc}
\hline Linguistic variable & Crisp value \\
\hline Equal importance (EI) & 1 \\
Weak importance (WI) & 3 \\
Moderate importance (MI) & 5 \\
Strong importance (SI) & 7 \\
Very strong importance (VS) & 9 \\
\hline
\end{tabular}

Table 4. Linguistic variables for the blue-green technologies importance

\begin{tabular}{|c|c|c|c|c|c|c|}
\hline & \multicolumn{6}{|c|}{ Economic criteria } \\
\hline & $\mathrm{C}_{11} / \mathrm{C}_{12}$ & $\mathrm{C}_{11} / \mathrm{C}_{13}$ & $\mathrm{C}_{11} / \mathrm{C}_{14}$ & $\mathrm{C}_{12} / \mathrm{C}_{13}$ & $\mathrm{C}_{12} / \mathrm{C}_{13}$ & $\mathrm{C}_{13} / \mathrm{C}_{14}$ \\
\hline E1 & EI & MI & SI & SI & MI & SI \\
\hline E2 & VS & SI & VS & VS & VS & VS \\
\hline E3 & MI & MI & SI & SI & WI & SI \\
\hline & $\mathrm{C}_{21} / \mathrm{C}_{22}$ & $\mathrm{C}_{21} / \mathrm{C}_{23}$ & $\mathrm{C}_{21} / \mathrm{C}_{24}$ & $\mathrm{C}_{22} / \mathrm{C}_{23}$ & $\mathrm{C}_{22} / \mathrm{C}_{23}$ & $\mathrm{C}_{23} / \mathrm{C}_{24}$ \\
\hline E1 & SI & MI & VS & VS & VS & SI \\
\hline E2 & VS & SI & VS & SI & VS & SI \\
\hline E3 & SI & SI & SI & VS & SI & SI \\
\hline & $\mathrm{C}_{31} / \mathrm{C}_{32}$ & $\mathrm{C}_{31} / \mathrm{C}_{33}$ & $\mathrm{C}_{31} / \mathrm{C}_{34}$ & $\mathrm{C}_{32} / \mathrm{C}_{33}$ & $\mathrm{C}_{32} / \mathrm{C}_{33}$ & $\mathrm{C}_{33} / \mathrm{C}_{34}$ \\
\hline E1 & MI & SI & SI & MI & MI & MI \\
\hline E2 & MI & WI & WI & SI & WI & VSI \\
\hline E3 & MI & SI & MI & MI & MI & M1 \\
\hline & $\mathrm{C}_{41} / \mathrm{C}_{42}$ & $\mathrm{C}_{41} / \mathrm{C}_{43}$ & $\mathrm{C}_{41} / \mathrm{C}_{44}$ & $\mathrm{C}_{41} / \mathrm{C}_{45}$ & $\mathrm{C}_{41} / \mathrm{C}_{46}$ & $\mathrm{C}_{42} / \mathrm{C}_{43}$ \\
\hline E1 & SI & MI & MI & MI & VS & VS \\
\hline E2 & VS & SI & VS & SI & VS & VS \\
\hline E3 & VS & SI & VS & SI & SI & SI \\
\hline
\end{tabular}


Journal of Digital Food, Energy \& Water Systems, 1 (1): 15-33, 2020

ISSN 2709-4529

(C) Centre for Cyber Physical Food, Energy \& Water Systems

\begin{tabular}{|l|l|l|l|l|l|l|}
\hline & $\mathrm{C}_{42} / \mathrm{C}_{44}$ & $\mathrm{C}_{42} / \mathrm{C}_{45}$ & $\mathrm{C}_{42} / \mathrm{C}_{46}$ & $\mathrm{C}_{43} / \mathrm{C}_{44}$ & $\mathrm{C}_{43} / \mathrm{C}_{45}$ & $\mathrm{C}_{43} / \mathrm{C}_{46}$ \\
\hline E1 & $\mathrm{SI}$ & $\mathrm{MI}$ & $\mathrm{VS}$ & $\mathrm{MI}$ & $\mathrm{SI}$ & $\mathrm{VS}$ \\
\hline E2 & $\mathrm{VS}$ & $\mathrm{VS}$ & $\mathrm{VI}$ & $\mathrm{SI}$ & $\mathrm{VS}$ & $\mathrm{VS}$ \\
\hline E3 & $\mathrm{MI}$ & $\mathrm{SI}$ & $\mathrm{SI}$ & $\mathrm{SI}$ & $\mathrm{MI}$ & $\mathrm{VS}$ \\
\hline & $\mathrm{C}_{44} / \mathrm{C}_{45}$ & $\mathrm{C}_{44} / \mathrm{C}_{45}$ & $\mathrm{C}_{45} / \mathrm{C}_{46}$ & & & \\
\hline E1 & MI & VS & VS & & & \\
\hline E2 & VS & VS & VS & & & \\
\hline E3 & VS & SI & VS & & & \\
\hline
\end{tabular}

Table 5. Best worst method results

\begin{tabular}{lcccccc}
\hline Economic & $\mathrm{C}_{11}$ & $\mathrm{C}_{12}$ & $\mathrm{C}_{13}$ & $\mathrm{C}_{14}$ & & \\
\hline 0.6730 & 0.46 & 0.37 & 0.13 & 0.04 & & \\
\hline Environmental & $\mathrm{C}_{31}$ & $\mathrm{C}_{32}$ & $\mathrm{C}_{33}$ & $\mathrm{C}_{34}$ \\
\hline 0.0647 & 0.49 & 0.36 & 0.10 & 0.05 & & \\
\hline Social & $\mathrm{C}_{41}$ & $\mathrm{C}_{42}$ & $\mathrm{C}_{43}$ & $\mathrm{C}_{44}$ & & \\
\hline 0.0528 & 0.53 & 0.27 & 0.13 & 0.07 & & $\mathrm{C}_{26}$ \\
\hline Technical & $\mathrm{C}_{21}$ & $\mathrm{C}_{22}$ & $\mathrm{C}_{23}$ & $\mathrm{C}_{24}$ & 0.03 & 0.03 \\
\hline 0.2088 & 0.31 & 0.37 & 0.21 & 0.03 & 0.05 & \\
\hline
\end{tabular}

Table 6 presents the linguistic terms used to evaluate technology appropriateness for the case study. Using these terms, Table 7 shows the experts' rating of the technologies' appropriateness for the case study.

Table 6. Linguistic terms for the technologies evaluation

\begin{tabular}{lclc}
\hline \multicolumn{2}{c}{ Benefit-based criterion } & \multicolumn{2}{c}{ Cost-based criterion } \\
\hline Linguistic terms & $\begin{array}{c}\text { Trapezoidal fuzzy } \\
\text { numbers }\end{array}$ & Linguistic terms & $\begin{array}{c}\text { Trapezoidal fuzzy } \\
\text { numbers }\end{array}$ \\
Very low & $0.0,0.1,0.2,0.3$ & Very low & $0.6,0.7,0.8,0.9$ \\
Low & $0.2,0.3,0.4,0.5$ & Low & $0.4,0.5,0.6,0.7$ \\
High & $0.4,0.5,0.6,0.7$ & High & $0.2,0.3,0.4,0.5$ \\
Very high & $0.6,0.7,0.8,0.9$ & Very high & $0.0,0.1,0.2,0.3$ \\
\hline
\end{tabular}


Journal of Digital Food, Energy \& Water Systems, 1 (1): 15-33, 2020 ISSN 2709-4529

(c) Centre for Cyber Physical Food, Energy \& Water Systems

Table 7. Linguistic values for the blue-green technologies

\begin{tabular}{|c|c|c|c|c|c|c|c|c|c|c|c|c|c|c|c|c|c|c|}
\hline & $\mathrm{C}_{11}$ & $\mathrm{C}_{12}$ & $\mathrm{C}_{13}$ & $\mathrm{C}_{14}$ & $\mathrm{C}_{21}$ & $\mathrm{C}_{22}$ & $\mathrm{C}_{23}$ & $\mathrm{C}_{24}$ & $\mathrm{C}_{31}$ & $\mathrm{C}_{32}$ & $\mathrm{C}_{33}$ & $\mathrm{C}_{34}$ & $\mathrm{C}_{41}$ & $\mathrm{C}_{42}$ & $\mathrm{C}_{43}$ & $\mathrm{C}_{44}$ & $\mathrm{C}_{45}$ & $\mathrm{C}_{46}$ \\
\hline \multicolumn{19}{|c|}{ E1 } \\
\hline A1 & $\mathrm{H}$ & $\mathrm{H}$ & $\mathrm{H}$ & VH & VH & $\mathrm{VH}$ & $\mathrm{H}$ & $\mathrm{H}$ & $\mathrm{VH}$ & $\mathrm{VH}$ & $\mathrm{L}$ & $\mathrm{H}$ & VH & $\mathrm{H}$ & $\mathrm{VH}$ & $\mathrm{H}$ & $\mathrm{H}$ & $\mathrm{VH}$ \\
\hline $\mathrm{A} 2$ & VH & VH & $\mathrm{H}$ & VH & $\mathrm{H}$ & $\mathrm{L}$ & $\mathrm{L}$ & $\mathrm{L}$ & $\mathrm{L}$ & $\mathrm{L}$ & $\mathrm{H}$ & $\mathrm{L}$ & VH & $\mathrm{H}$ & $\mathrm{H}$ & $\mathrm{H}$ & VH & $\mathrm{VH}$ \\
\hline A3 & VH & VH & $\mathrm{H}$ & $\mathrm{H}$ & $\mathrm{H}$ & $\mathrm{L}$ & $\mathrm{L}$ & $\mathrm{H}$ & $\mathrm{H}$ & $\mathrm{L}$ & $\mathrm{L}$ & $\mathrm{H}$ & $\mathrm{H}$ & $\mathrm{H}$ & $\mathrm{H}$ & $\mathrm{H}$ & L & $\mathrm{VH}$ \\
\hline A4 & VL & $\mathrm{H}$ & $\mathrm{L}$ & $\mathrm{H}$ & $\mathrm{H}$ & $\mathrm{H}$ & $\mathrm{H}$ & $\mathrm{L}$ & $\mathrm{H}$ & $\mathrm{H}$ & $\mathrm{L}$ & $\mathrm{H}$ & VH & $\mathrm{H}$ & $\mathrm{H}$ & $\mathrm{L}$ & $\mathrm{L}$ & $\mathrm{VH}$ \\
\hline A5 & $\mathrm{H}$ & $\mathrm{H}$ & $\mathrm{L}$ & $\mathrm{L}$ & $\mathrm{H}$ & $\mathrm{H}$ & $\mathrm{L}$ & $\mathrm{L}$ & $\mathrm{H}$ & $\mathrm{H}$ & VL & $\mathrm{L}$ & VH & $\mathrm{H}$ & $\mathrm{H}$ & $\mathrm{L}$ & $\mathrm{H}$ & $\mathrm{VH}$ \\
\hline A6 & VH & $\mathrm{L}$ & $\mathrm{H}$ & $\mathrm{L}$ & $\mathrm{H}$ & $\mathrm{L}$ & VL & $\mathrm{L}$ & VH & $\mathrm{H}$ & $\mathrm{L}$ & $\mathrm{L}$ & VH & VH & $\mathrm{VH}$ & VH & $\mathrm{L}$ & $\mathrm{VH}$ \\
\hline \multicolumn{19}{|c|}{ E2 } \\
\hline A1 & $\mathrm{VH}$ & $\mathrm{H}$ & $\mathrm{L}$ & $\mathrm{VH}$ & $\mathrm{VH}$ & $\mathrm{VH}$ & $\mathrm{H}$ & $\mathrm{VH}$ & $\mathrm{H}$ & $\mathrm{H}$ & $\mathrm{VH}$ & $\mathrm{H}$ & $\mathrm{L}$ & $\mathrm{VH}$ & $\mathrm{H}$ & $\mathrm{VH}$ & $\mathrm{L}$ & $\mathrm{VH}$ \\
\hline A2 & VH & $\mathrm{VH}$ & $\mathrm{H}$ & VH & $\mathrm{H}$ & $\mathrm{H}$ & $\mathrm{H}$ & VH & $\mathrm{H}$ & $\mathrm{H}$ & VH & $\mathrm{H}$ & $\mathrm{H}$ & $\mathrm{H}$ & $\mathrm{L}$ & $\mathrm{H}$ & VH & $\mathrm{H}$ \\
\hline A3 & VH & $\mathrm{L}$ & $\mathrm{L}$ & VH & VH & $\mathrm{H}$ & $\mathrm{L}$ & $\mathrm{H}$ & $\mathrm{H}$ & $\mathrm{H}$ & $\mathrm{L}$ & VH & $\mathrm{H}$ & $\mathrm{H}$ & $\mathrm{H}$ & $\mathrm{H}$ & $\mathrm{L}$ & $\mathrm{H}$ \\
\hline A4 & $\mathrm{H}$ & $\mathrm{H}$ & $\mathrm{L}$ & VH & $\mathrm{L}$ & $\mathrm{H}$ & $\mathrm{L}$ & $\mathrm{H}$ & $\mathrm{L}$ & VL & $\mathrm{H}$ & $\mathrm{H}$ & $\mathrm{L}$ & $\mathrm{L}$ & $\mathrm{H}$ & VH & $\mathrm{H}$ & $\mathrm{L}$ \\
\hline A5 & VH & $\mathrm{H}$ & $\mathrm{L}$ & VH & $\mathrm{L}$ & $\mathrm{H}$ & $\mathrm{L}$ & $\mathrm{H}$ & $\mathrm{H}$ & $\mathrm{L}$ & $\mathrm{L}$ & $\mathrm{H}$ & VL & $\mathrm{L}$ & VL & $\mathrm{L}$ & $\mathrm{L}$ & $\mathrm{H}$ \\
\hline A6 & $\mathrm{H}$ & $\mathrm{L}$ & $\mathrm{H}$ & $\mathrm{H}$ & $\mathrm{H}$ & $\mathrm{VH}$ & $\mathrm{H}$ & $\mathrm{H}$ & $\mathrm{VH}$ & $\mathrm{VH}$ & $\mathrm{H}$ & $\mathrm{H}$ & $\mathrm{H}$ & $\mathrm{H}$ & $\mathrm{H}$ & $\mathrm{H}$ & VL & $\mathrm{H}$ \\
\hline \multicolumn{19}{|c|}{ E3 } \\
\hline A1 & $\mathrm{H}$ & $\mathrm{H}$ & $\mathrm{VH}$ & $\mathrm{H}$ & $\mathrm{H}$ & $\mathrm{H}$ & $\mathrm{H}$ & $\mathrm{H}$ & $\mathrm{H}$ & $\mathrm{H}$ & $\mathrm{VH}$ & $\mathrm{H}$ & $\mathrm{VH}$ & $\mathrm{H}$ & $\mathrm{H}$ & $\mathrm{VH}$ & $\mathrm{H}$ & $\mathrm{VH}$ \\
\hline A2 & VH & $\mathrm{H}$ & $\mathrm{H}$ & $\mathrm{H}$ & $\mathrm{L}$ & $\mathrm{H}$ & $\mathrm{L}$ & $\mathrm{H}$ & $\mathrm{L}$ & $\mathrm{L}$ & $\mathrm{L}$ & VH & $\mathrm{H}$ & $\mathrm{H}$ & $\mathrm{H}$ & $\mathrm{H}$ & $\mathrm{L}$ & $\mathrm{H}$ \\
\hline A3 & $\mathrm{H}$ & $\mathrm{H}$ & $\mathrm{L}$ & $\mathrm{L}$ & $\mathrm{H}$ & $\mathrm{H}$ & $\mathrm{H}$ & $\mathrm{H}$ & $\mathrm{H}$ & $\mathrm{H}$ & $\mathrm{L}$ & $\mathrm{H}$ & $\mathrm{H}$ & $\mathrm{H}$ & $\mathrm{L}$ & $\mathrm{L}$ & $\mathrm{L}$ & $\mathrm{L}$ \\
\hline A4 & $\mathrm{L}$ & $\mathrm{H}$ & $\mathrm{L}$ & $\mathrm{L}$ & $\mathrm{L}$ & $\mathrm{L}$ & $\mathrm{L}$ & $\mathrm{L}$ & $\mathrm{H}$ & $\mathrm{L}$ & VL & $\mathrm{L}$ & $\mathrm{L}$ & $\mathrm{H}$ & $\mathrm{H}$ & $\mathrm{L}$ & $\mathrm{L}$ & $\mathrm{H}$ \\
\hline A5 & $\mathrm{H}$ & $\mathrm{L}$ & $\mathrm{VH}$ & $\mathrm{H}$ & $\mathrm{L}$ & $\mathrm{L}$ & $\mathrm{H}$ & $\mathrm{L}$ & $\mathrm{L}$ & $\mathrm{H}$ & $\mathrm{L}$ & $\mathrm{H}$ & $\mathrm{H}$ & $\mathrm{H}$ & $\mathrm{L}$ & VH & $\mathrm{H}$ & $\mathrm{L}$ \\
\hline A6 & $\mathrm{L}$ & VH & $\mathrm{L}$ & $\mathrm{L}$ & $\mathrm{H}$ & $\mathrm{H}$ & $\mathrm{L}$ & $\mathrm{H}$ & $\mathrm{L}$ & $\mathrm{H}$ & $\mathrm{L}$ & VH & $\mathrm{L}$ & $\mathrm{H}$ & $\mathrm{L}$ & $\mathrm{H}$ & $\mathrm{L}$ & $\mathrm{L}$ \\
\hline
\end{tabular}


Using Equations (8) to (11), the linguistic values in Table 7 were converted and aggregated into fuzzy values (Table 8). Equation (12) is used to generate the weighted aggregated values for the technologies' based on the information tables 5 and 8 . Table 9 presents the results obtained for these values. This study used the concept of optimistic (a), pessimistic (m) and most likely design requirements (b) to generate the design requirements (Table 10). It considered Expert 1 has an optimist, Expert 2 has a realist, and Expert 3 has a pessimist.

$D R=\frac{a+4 m+b}{6}$

Equation (13) was used to generate the common requirements for the technologies using the information in tables 9 and 10. Furthermore, Equation (14) was used to compute the system requirements of the technologies. Table 11 presents the results for the technologies common and system requirements. Equation (15) was used to calculate the technologies' probabilities of meeting the design requirements. This study used Equation (16) to compute the technologies' information contents (Table 12). 
Table 8. Aggregated values of the technologies

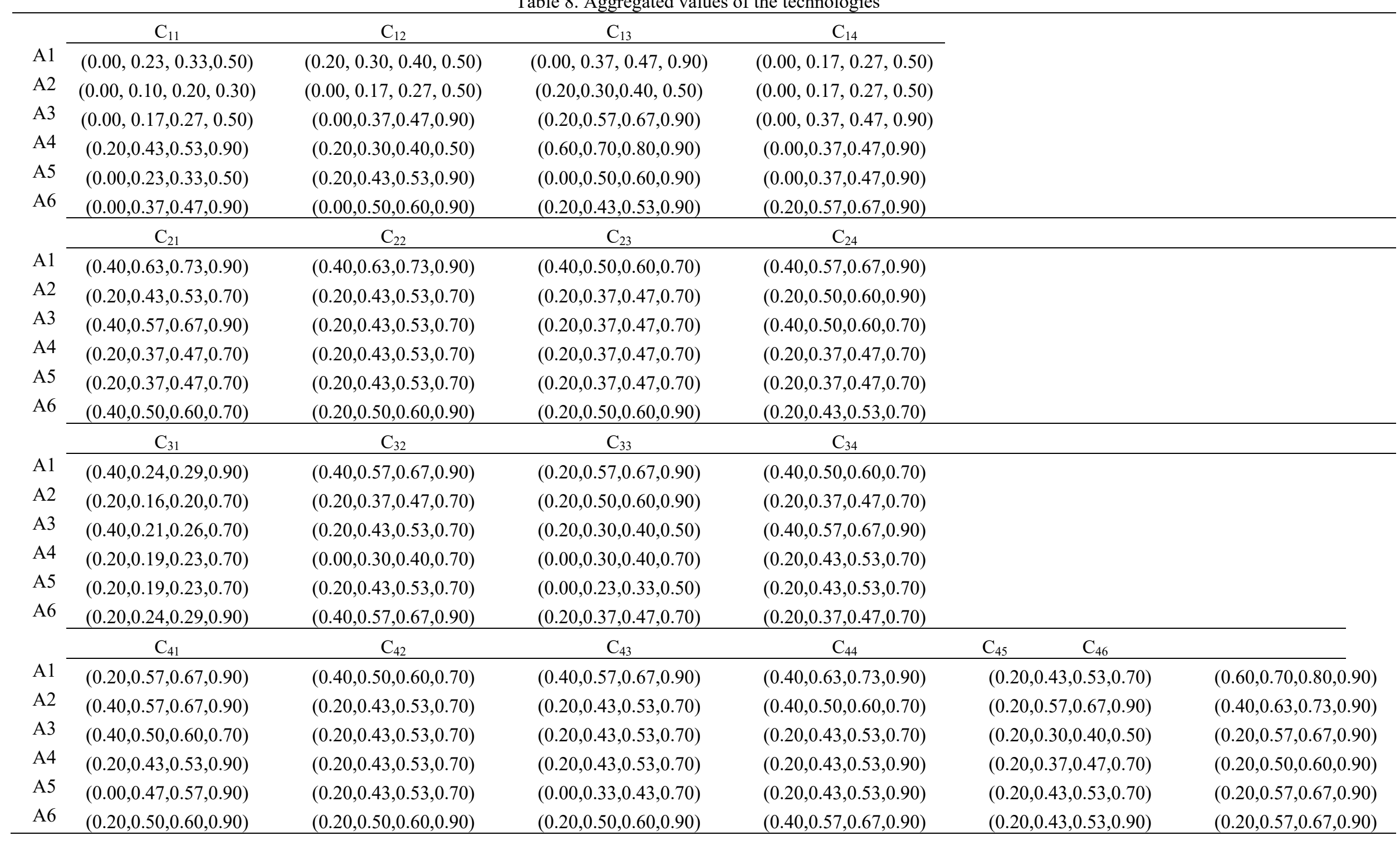


Table 9. Weighted aggregated values of the technologies

\begin{tabular}{|c|c|c|c|c|c|c|}
\hline & $\mathrm{C}_{11}$ & $\mathrm{C}_{12}$ & $\mathrm{C}_{13}$ & $\mathrm{C}_{14}$ & & \\
\hline A1 & $(0.11,0.23,0.33,0.58)$ & $(0.24,0.30,0.40,0.54)$ & $(0.05,0.37,0.47,0.96)$ & $(0.01,0.17,0.27,0.51)$ & & \\
\hline A2 & $(0.05,0.10,0.20,0.35)$ & $(0.06,0.17,0.27,0.59)$ & $(0.21,0.30,0.40,0.51)$ & $(0.01,0.17,0.27,0.51)$ & & \\
\hline A3 & $(0.08,0.17,0.27,0.61)$ & $(0.14,0.37,0.47,1.00)$ & $(0.25,0.57,0.67,0.93)$ & $(0.01,0.37,0.47,0.92)$ & & \\
\hline A4 & $(0.31,0.43,0.53,1.00)$ & $(0.24,0.30,0.40,0.54)$ & $(0.61,0.70,0.80,0.91)$ & $(0.01,0.37,0.47,0.92)$ & & \\
\hline A5 & $(0.11,0.23,0.33,0.58)$ & $(0.29,0.43,0.53,1.00)$ & $(0.07,0.50,0.60,0.94)$ & $(0.01,0.37,0.47,0.92)$ & & \\
\hline \multirow[t]{2}{*}{ A6 } & $(0.17,0.37,0.47,1.00)$ & $(0.19,0.50,0.60,1.00)$ & $(0.23,0.43,0.53,0.95)$ & $(0.21,0.57,0.67,0.91)$ & & \\
\hline & $\mathrm{C}_{21}$ & $\mathrm{C}_{22}$ & $\mathrm{C}_{23}$ & $\mathrm{C}_{24}$ & & \\
\hline A1 & $(0.51,0.63,0.73,0.98)$ & $(0.48,0.63,0.73,0.96)$ & $(0.41,0.50,0.60,0.71)$ & $(0.41,0.57,0.67,0.91)$ & & \\
\hline A2 & $(0.31,0.43,0.53,0.78)$ & $(0.28,0.43,0.53,0.76)$ & $(0.22,0.37,0.47,0.72)$ & $(0.22,0.50,0.60,0.92)$ & & \\
\hline A3 & $(0.48,0.57,0.67,1.00)$ & $(0.28,0.43,0.53,0.76)$ & $(0.22,0.37,0.47,0.72)$ & $(0.41,0.50,0.60,0.71)$ & & \\
\hline A4 & $(0.28,0.37,0.47,0.81)$ & $(0.28,0.43,0.53,0.76)$ & $(0.22,0.37,0.47,0.72)$ & $(0.21,0.37,0.47,0.71)$ & & \\
\hline A5 & $(0.28,0.37,0.47,0.81)$ & $(0.28,0.43,0.53,0.76)$ & $(0.22,0.37,0.47,0.72)$ & $(0.21,0.37,0.47,0.71)$ & & \\
\hline \multirow[t]{2}{*}{ A6 } & $(0.45,0.50,0.60,0.75)$ & $(0.31,0.50,0.60,1.00)$ & $(0.23,0.50,0.60,0.93)$ & $(0.21,0.43,0.53,0.71)$ & & \\
\hline & $\mathrm{C}_{31}$ & $\mathrm{C}_{32}$ & $\mathrm{C}_{33}$ & $\mathrm{C}_{34}$ & & \\
\hline A1 & $(0.32,0.24,0.29,1.23)$ & $(0.45,0.57,0.67,0.96)$ & $(0.25,0.57,0.67,0.93)$ & $(0.41,0.50,0.60,0.71)$ & & \\
\hline A2 & $(0.18,0.16,0.20,0.97)$ & $(0.25,0.37,0.47,0.76)$ & $(0.24,0.50,0.60,0.94)$ & $(0.21,0.37,0.47,0.72)$ & & \\
\hline A3 & $(0.30,0.21,0.26,0.93)$ & $(0.26,0.43,0.53,0.75)$ & $(0.21,0.30,0.40,0.51)$ & $(0.41,0.57,0.67,0.92)$ & & \\
\hline A4 & $(0.19,0.19,0.23,0.95)$ & $(0.08,0.30,0.40,0.78)$ & $(0.04,0.30,0.40,0.74)$ & $(0.22,0.43,0.53,0.71)$ & & \\
\hline A5 & $(0.19,0.19,0.23,0.95)$ & $(0.26,0.43,0.53,0.75)$ & $(0.03,0.23,0.33,0.52)$ & $(0.22,0.43,0.53,0.71)$ & & \\
\hline \multirow[t]{2}{*}{ A6 } & $(0.22,0.24,0.29,1.23)$ & $(0.45,0.57,0.67,0.96)$ & $(0.22,0.37,0.47,0.73)$ & $(0.21,0.37,0.47,0.72)$ & & \\
\hline & $\mathrm{C}_{41}$ & $\mathrm{C}_{42}$ & $\mathrm{C}_{43}$ & $\mathrm{C}_{44}$ & $\mathrm{C}_{45}$ & $\mathrm{C}_{46}$ \\
\hline A1 & $(0.31,0.57,0.67,0.97)$ & $(0.44,0.50,0.60,0.74)$ & $(0.44,0.57,0.67,0.95)$ & $(0.41,0.63,0.73,0.91)$ & $(0.21,0.43,0.53,0.71)$ & $(0.60,0.70,0.80,0.90)$ \\
\hline A2 & $(0.45,0.57,0.67,0.97)$ & $(0.29,0.43,0.53,0.76)$ & $(0.25,0.43,0.53,0.74)$ & $(0.40,0.50,0.60,0.70)$ & $(0.22,0.57,0.67,0.91)$ & $(0.41,0.63,0.73,0.91)$ \\
\hline A3 & $(0.43,0.50,0.60,0.73)$ & $(0.29,0.43,0.53,0.76)$ & $(0.25,0.43,0.53,0.74)$ & $(0.21,0.43,0.53,0.71)$ & $(0.21,0.30,0.40,0.51)$ & $(0.21,0.57,0.67,0.91)$ \\
\hline A4 & $(0.27,0.43,0.53,1.00)$ & $(0.29,0.43,0.53,0.76)$ & $(0.25,0.43,0.53,0.74)$ & $(0.21,0.43,0.53,0.91)$ & $(0.21,0.37,0.47,0.71)$ & $(0.21,0.50,0.60,0.91)$ \\
\hline A5 & $(0.14,0.47,0.57,1.00)$ & $(0.29,0.43,0.53,0.76)$ & $(0.07,0.33,0.43,0.76)$ & $(0.21,0.43,0.53,0.91)$ & $(0.21,0.43,0.53,0.71)$ & $(0.21,0.57,0.67,0.91)$ \\
\hline A6 & $(0.29,0.50,0.60,0.99)$ & $(0.31,0.50,0.60,1.00)$ & $(0.26,0.50,0.60,0.96)$ & $(0.41,0.57,0.67,0.91)$ & $(0.21,0.43,0.53,0.92)$ & $(0.21,0.57,0.67,0.91)$ \\
\hline
\end{tabular}


Table 10. Design requirements

\begin{tabular}{lc}
\hline & Trapezoidal fuzzy numbers \\
\hline $\mathrm{C}_{11}$ & $(0.27,0.33,0.43,0.57)$ \\
$\mathrm{C}_{12}$ & $(0.28,0.34,0.44,0.58)$ \\
$\mathrm{C}_{13}$ & $(0.37,0.46,0.56,0.67)$ \\
$\mathrm{C}_{14}$ & $(0.32,0.42,0.52,0.62)$ \\
& \\
$\mathrm{C}_{21}$ & $(0.34,0.44,0.54,0.64)$ \\
$\mathrm{C}_{22}$ & $(0.36,0.46,0.56,0.66)$ \\
$\mathrm{C}_{23}$ & $(0.31,0.41,0.51,0.61)$ \\
$\mathrm{C}_{24}$ & $(0.34,0.44,0.54,0.64)$ \\
$\mathrm{C}_{31}$ & \\
$\mathrm{C}_{32}$ & $(0.34,0.44,0.54,0.64)$ \\
$\mathrm{C}_{33}$ & $(0.34,0.44,0.54,0.64)$ \\
$\mathrm{C}_{34}$ & $(0.26,0.36,0.46,0.56)$ \\
$\mathrm{C}_{41}$ & $(0.41,0.51,0.61,0.71)$ \\
$\mathrm{C}_{42}$ & \\
$\mathrm{C}_{43}$ & $(0.33,0.42,0.50,0.59)$ \\
$\mathrm{C}_{44}$ & $(0.29,0.38,0.46,0.54)$ \\
$\mathrm{C}_{45}$ & $(0.26,0.34,0.43,0.51)$ \\
\hline
\end{tabular}

Table 11. The alternatives' common and system areas

\begin{tabular}{|c|c|c|c|c|c|c|c|c|c|c|c|c|}
\hline & A1 & $\mathrm{A} 2$ & A3 & A4 & A5 & A6 & A1 & $\mathrm{A} 2$ & A3 & A4 & A5 & A6 \\
\hline & \multicolumn{6}{|c|}{ Common area } & \multicolumn{6}{|c|}{ System area } \\
\hline \multicolumn{13}{|c|}{ Economic } \\
\hline $\mathrm{C}_{11}$ & 0.025 & 0.002 & 0.004 & 0.225 & 0.025 & 0.144 & 0.320 & 0.180 & 0.300 & 0.610 & 0.320 & 0.560 \\
\hline $\mathrm{C}_{12}$ & 0.064 & 0.002 & 0.132 & 0.064 & 0.210 & 0.324 & 0.370 & 0.280 & 0.530 & 0.370 & 0.600 & 0.580 \\
\hline $\mathrm{C}_{13}$ & 0.030 & 0.004 & 0.240 & 0.484 & 0.144 & 0.072 & 0.470 & 0.360 & 0.600 & 0.760 & 0.520 & 0.550 \\
\hline $\mathrm{C}_{14}$ & 0.006 & 0.006 & 0.056 & 0.056 & 0.056 & 0.306 & 0.240 & 0.240 & 0.450 & 0.450 & 0.450 & 0.580 \\
\hline \multicolumn{13}{|c|}{ Environmental } \\
\hline $\mathrm{C}_{21}$ & 0.380 & 0.090 & 0.272 & 0.042 & 0.042 & 0.169 & 0.720 & 0.520 & 0.700 & 0.500 & 0.500 & 0.580 \\
\hline $\mathrm{C}_{22}$ & 0.342 & 0.072 & 0.072 & 0.072 & 0.072 & 0.144 & 0.700 & 0.500 & 0.500 & 0.500 & 0.500 & 0.620 \\
\hline $\mathrm{C}_{23}$ & 0.210 & 0.064 & 0.064 & 0.064 & 0.064 & 0.210 & 0.560 & 0.450 & 0.450 & 0.450 & 0.450 & 0.570 \\
\hline $\mathrm{C}_{24}$ & 0.272 & 0.169 & 0.169 & 0.042 & 0.042 & 0.090 & 0.640 & 0.560 & 0.560 & 0.440 & 0.440 & 0.470 \\
\hline \multicolumn{13}{|c|}{ Social } \\
\hline $\mathrm{C}_{31}$ & 0.008 & 0.070 & 0.021 & 0.043 & 0.043 & 0.008 & 0.600 & 0.440 & 0.480 & 0.440 & 0.440 & 0.560 \\
\hline $\mathrm{C}_{32}$ & 0.272 & 0.042 & 0.090 & 0.009 & 0.090 & 0.272 & 0.670 & 0.470 & 0.500 & 0.400 & 0.500 & 0.670 \\
\hline $\mathrm{C}_{33}$ & 0.420 & 0.289 & 0.049 & 0.049 & 0.012 & 0.110 & 0.600 & 0.580 & 0.360 & 0.380 & 0.280 & 0.450 \\
\hline $\mathrm{C}_{34}$ & 1.960 & 0.563 & 3.063 & 1.103 & 1.103 & 0.563 & 0.560 & 0.560 & 0.650 & 0.470 & 0.470 & 0.560 \\
\hline \multicolumn{13}{|c|}{ Technical } \\
\hline $\mathrm{C}_{41}$ & 0.304 & 0.304 & 0.192 & 0.105 & 0.152 & 0.192 & 0.630 & 0.680 & 0.570 & 0.580 & 0.550 & 0.610 \\
\hline
\end{tabular}


Journal of Digital Food, Energy \& Water Systems, 1 (1): 15-33, 2020

ISSN 2709-4529

(C) Centre for Cyber Physical Food, Energy \& Water Systems

\begin{tabular}{lllllllllllll}
\hline $\mathrm{C}_{42}$ & 0.253 & 0.152 & 0.152 & 0.152 & 0.152 & 0.253 & 0.570 & 0.510 & 0.510 & 0.510 & 0.510 & 0.620 \\
$\mathrm{C}_{43}$ & 0.467 & 0.203 & 0.203 & 0.203 & 0.080 & 0.321 & 0.670 & 0.490 & 0.490 & 0.490 & 0.400 & 0.590 \\
$\mathrm{C}_{44}$ & 0.444 & 0.203 & 0.111 & 0.111 & 0.111 & 0.321 & 0.670 & 0.550 & 0.470 & 0.530 & 0.530 & 0.640 \\
$\mathrm{C}_{45}$ & 0.218 & 0.490 & 0.063 & 0.134 & 0.218 & 0.218 & 0.470 & 0.590 & 0.360 & 0.440 & 0.470 & 0.530 \\
$\mathrm{C}_{46}$ & 0.538 & 0.380 & 0.267 & 0.160 & 0.267 & 0.267 & 0.750 & 0.670 & 0.580 & 0.560 & 0.580 & 0.580 \\
\hline
\end{tabular}

Table 12. The alternatives' information contents

\begin{tabular}{|c|c|c|c|c|c|c|}
\hline & A1 & $\mathrm{A} 2$ & A3 & A4 & A5 & A6 \\
\hline \multicolumn{7}{|c|}{ Economic } \\
\hline $\mathrm{C}_{11}$ & 0.272 & 0.158 & 0.161 & 0.695 & 0.272 & 0.510 \\
\hline $\mathrm{C}_{12}$ & 0.395 & 0.144 & 0.499 & 0.395 & 0.661 & 1.190 \\
\hline $\mathrm{C}_{13}$ & 0.253 & 0.154 & 0.757 & 1.536 & 0.540 & 0.341 \\
\hline $\mathrm{C}_{14}$ & 0.190 & 0.190 & 0.333 & 0.333 & 0.333 & 1.085 \\
\hline Total & 1.110 & 0.646 & 1.751 & 2.959 & 1.806 & 3.128 \\
\hline \multicolumn{7}{|c|}{ Environmental } \\
\hline $\mathrm{C}_{21}$ & 1.086 & 0.396 & 0.734 & 0.281 & 0.281 & 0.562 \\
\hline $\mathrm{C}_{22}$ & 0.969 & 0.358 & 0.358 & 0.358 & 0.358 & 0.475 \\
\hline $\mathrm{C}_{23}$ & 0.708 & 0.355 & 0.355 & 0.355 & 0.355 & 0.695 \\
\hline $\mathrm{C}_{24}$ & 0.811 & 0.579 & 0.579 & 0.296 & 0.296 & 0.420 \\
\hline Total & 3.573 & 1.688 & 2.026 & 1.290 & 1.290 & 2.152 \\
\hline \multicolumn{7}{|c|}{ Social } \\
\hline $\mathrm{C}_{31}$ & 0.162 & 0.377 & 0.223 & 0.299 & 0.299 & 0.165 \\
\hline $\mathrm{C}_{32}$ & 0.770 & 0.288 & 0.405 & 0.183 & 0.405 & 0.770 \\
\hline $\mathrm{C}_{33}$ & 1.947 & 0.995 & 0.348 & 0.338 & 0.222 & 0.493 \\
\hline $\mathrm{C}_{34}$ & 1.426 & 2.039 & 0.25 & 3.419 & 3.525 & 1.426 \\
\hline Total & 4.305 & 3.699 & 1.226 & 4.239 & 4.451 & 2.854 \\
\hline & \multicolumn{6}{|c|}{ Technical } \\
\hline $\mathrm{C}_{41}$ & 0.952 & 0.862 & 0.637 & 0.406 & 0.538 & 0.599 \\
\hline $\mathrm{C}_{42}$ & 0.853 & 0.571 & 0.571 & 0.571 & 0.571 & 0.773 \\
\hline $\mathrm{C}_{43}$ & 1.920 & 0.784 & 0.784 & 0.784 & 0.432 & 1.139 \\
\hline $\mathrm{C}_{44}$ & 1.689 & 0.694 & 0.481 & 0.444 & 0.444 & 1.005 \\
\hline $\mathrm{C}_{45}$ & 0.901 & 3.732 & 0.396 & 0.585 & 0.901 & 0.779 \\
\hline $\mathrm{C}_{46}$ & 2.084 & 1.224 & 0.893 & 0.553 & 0.893 & 0.893 \\
\hline Total & 8.398 & 7.867 & 3.762 & 3.343 & 3.779 & 5.189 \\
\hline
\end{tabular}

Aggregation of the information in Table 12 was carried out using GRA. During this process, an identification coefficient of 0.5 to analyse the blue-green technologies [32]. Equation (20) was used to normalised the information in Table 13 because of the FA method rank output in terms of the smallerthe-better. Table 14 presents the GRA output for the blue-green technology problem. 
Table 13. Normalised decision-matrix for GRA application

\begin{tabular}{lcccc}
\hline & $\mathrm{C} 1$ & $\mathrm{C} 2$ & $\mathrm{C} 3$ & $\mathrm{C} 4$ \\
$\mathrm{~A} 1$ & 0.187 & 1.000 & 1.000 & 0.955 \\
$\mathrm{~A} 2$ & 0.000 & 0.895 & 0.174 & 0.767 \\
$\mathrm{~A} 3$ & 0.445 & 0.083 & 0.322 & 0.000 \\
$\mathrm{~A} 4$ & 0.932 & 0.000 & 0.000 & 0.934 \\
$\mathrm{~A} 5$ & 0.467 & 0.086 & 0.000 & 1.000 \\
$\mathrm{~A} 6$ & 1.000 & 0.365 & 0.378 & 0.505 \\
\hline
\end{tabular}

Table 14. GRA outputs for the blue-green technology problem

\begin{tabular}{lccccc}
\hline & $\mathrm{C} 1$ & $\mathrm{C} 2$ & $\mathrm{C} 3$ & $\mathrm{C} 4$ & Grade \\
$\mathrm{A} 1$ & 0.647 & 0.308 & 0.298 & 0.211 & 0.132 \\
$\mathrm{~A} 2$ & 1.130 & 0.336 & 0.878 & 0.251 & 0.225 \\
$\mathrm{~A} 3$ & 0.406 & 1.156 & 0.651 & 1.019 & 0.153 \\
$\mathrm{~A} 4$ & 0.239 & 1.539 & 1.490 & 0.215 & 0.148 \\
$\mathrm{~A} 5$ & 0.394 & 1.144 & 1.490 & 0.204 & 0.153 \\
$\mathrm{~A} 6$ & 0.226 & 0.626 & 0.593 & 0.338 & 0.085 \\
\hline
\end{tabular}

\section{Discussion of Results}

The best-worst results for the current problem showed that the techno-economic criteria contributed about $88.18 \%$ to the ranking of blue-green technology. In terms of the economic sub-criteria, the contributions of $\mathrm{C}_{11}$ and $\mathrm{C}_{12}$ to the technology selection was about $83 \%$ (Table 5). In comparison, Table 5 showed that $\mathrm{C}_{21}$ and $\mathrm{C}_{22}$ contributed about $85 \%$ to the technology selection. The contributions of $\mathrm{C}_{31}$ and $\mathrm{C}_{32}$ to the selection problem in terms of social criterion were about $8 \%$. This study observed that at least three technical sub-criteria account for about $80 \%$ of the sub-criteria that affect these technologies selection. Another unique feature of the technical sub-criteria is that the first criterion was not ranked as the most important criterion; instead, it was $\mathrm{C}_{42}$ that was the most crucial criterion.

Figure 3 shows the selected blue-green technologies rank in terms of the FA and GRA methods. When the FA method results aggregated with the GRA method, the most and least suitable technology for the case study was A1 and A6, respectively; these rankings are consistent with the economic criteria ranks for the case study. The GRA method results showed that the suitability of A3 and A5 for the case study area is the same (Figure 4). The technical and environmental criteria FA method results showed A4 is the most suitable technology, while A1 is the least suitable technology (Figure 4). In terms of the social criterion, the most suitable technology is A3, while A5 is the least suitable technology.

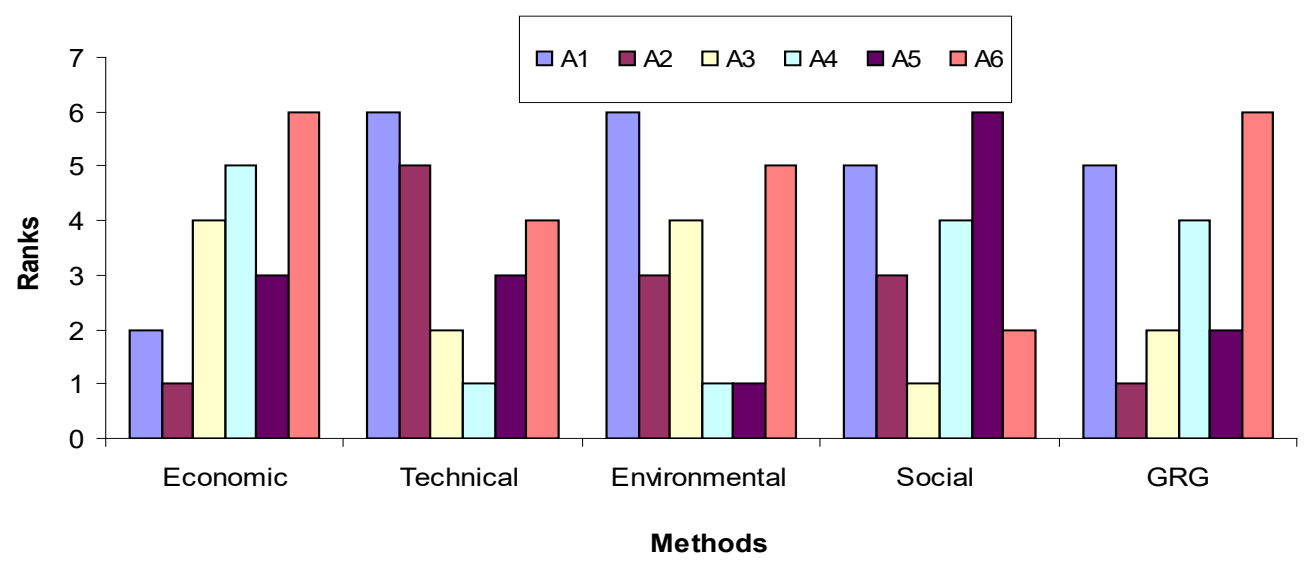

Figure 4: Comparison of different methods for blue-green technology ranking 


\section{Policy implications}

This study outputs have several policy implications to FRM problem in developing countries. First, it has been able to establish that MCDM tools can be used to provide bounded-rational on the most suitable technology for flood control. With this knowledge, corporate responsibility can be taken whenever a technology fails. This identification process will absolve a particular person from bearing the effect of a policy failure. Second, policy failure can be tacked from strategy and tactical level. When considering a strategic level to policy failure, policy-makers can trouble-shoot failure by considering economic, social, environmental and technical requirements for this technology selection. On the other hand, policy failure can be traced to the sub-criteria that constitute each of the criteria, as mentioned earlier at a strategic level.

\section{Conclusion}

This study has developed a framework for blue-green technology selection. It used a multi-criterion modelling approach to developed the framework. Sustainability criteria were embedded into the framework to account for stakeholders' requirement during the selection process. Lekki, a community in Lagos, Nigeria, was used as a case study for the framework evaluation. Based on three experts' responses about six blue-green technologies, which include bypass floodway, rainwater and floodwater harvesting, and porous pavement, the most and least suitable technologies were identified for the case study. The framework identified rainwater and floodwater harvesting as the most suitable blue-green technologies for a community. It also identified a bypass floodway as the least suitable blue-green technologies for a community.

The use of a best-worst method to address the problem of optimal weight determination for criteria is a contribution of this study. Another contribution of the current study is the fuzzy axiomatic method to incorporate decision-makers' preference into blue-green technology selection. Also, this study contributed to the use of optimistic, pessimistic and most likely design requirements approach to fuzzy axiomatic method application as another contribution to FRM. Also, it applied GRA to FRM problem as a contribution to FRM. One of the limitations of this study is that it did not consider the political and institutional constraints that affect blue-green technology selection. This study, therefore, expects future studies to incorporate these factors into blue-green technology analysis.

\section{Acknowledgements}

Funding was not available for this research

\section{Conflict of Interest}

The authors declare that there is no conflict of interest.

\section{References}

[1] J. Lamond, O. Adekola, I. Adelekan, B. Eze, F. Ujoh, Information for adaptation and response to flooding, multi-stakeholder perspectives in Nigeria, Climate 7 (4) (2019) 46.

[2] X. Cheng, Changes of flood control situations and adjustments of flood management strategies in China, Water international 30, (1) (2005): 108-113.

[3] A. J. Echendu, The impact of flooding on Nigeria's sustainable development goals (SDGs), Ecosystem Health and Sustainability 6 (1) (2020) 1791735.

[4] E. Lawson, C. Thorne, S. Ahilan, D. Allen, S. Arthur, G. Everett, R. Fenner, V. Glenis, D. Guan, L. Hoang, C. Kilsby, Delivering and evaluating the multiple flood risk benefits in blue-green cities: An interdisciplinary approach, WIT Transactions on Ecology and the Environment, 184 (2014) 113-124.

[5] G. Veilleux, T. Potisat, D. Pezim, C. Ribback, J. Ling, A. Krysztofiński, A. Ahmed, J. Papenheim, A.M. Pineda, S. Sembian, S. Chucherd, Techno-economic analysis of microgrid projects for rural electrification: A systematic approach to the redesign of Koh Jik off-grid case study, Energy for Sustainable Development, 54 (2020) 1-13.

[6] D.L. Hegger, P.P. Driessen, M. Wiering, H.F. Van Rijswick, Z.W. Kundzewicz, P. Matczak, A. Crabbé, G.T. Raadgever, M.H. Bakker, S.J. Priest, C., Larrue, Toward more flood resilience: Is a diversification of flood risk management strategies the way forward? Ecology and Society, 21(4) (2016).

[7] M. Vis, F. Klijn, K. M. De Bruijn, M. Van Buuren, Resilience strategies for flood risk management in the Netherlands, International Journal of River Basin Management 1 (1) (2003) 33-40. 
[8] L. McFadden, E. Penning-Rowsell, S. Tapsell, Strategic coastal flood-risk management in practice: Actors' perspectives on the integration of flood risk management in London and the Thames Estuary, Ocean Coast. Ocean and Coastal Management 52 (12) (2009) 636-645.

[9] N. Doorn, Rationality in flood risk management: the limitations of probabilistic risk assessment in the design and selection of flood protection strategies, Journal of Flood Risk Management 7 (3) (2014):230-238.

[10] L. Solin, M. S. Madajová, L. Michaleje, Vulnerability assessment of households and its possible reflection in flood risk management: The case of the upper Myjava basin, Slovakia, International Journal of Disaster Risk Reduction 28 (2018) 640-652.

[11] E. Lawson, C. Thorne, N. Wright, R, Fenner, S. Arthur, J. Lamond, C. Kilsby, J. Mant, L. Smith, S. Ahilan, D. Allen, Evaluating the multiple benefits of a Blue-Green Vision for urban surface water management. In UDG Autumn Conference and Exhibition 2015. Leeds, 2015.

[12] E. E. Koks, B. Jongman, T. G. Husby, W. J. W. Botzen, Combining hazard, exposure and social vulnerability to provide lessons for flood risk management, Environmental Science and Policy 47 (2015) 42-52.

[13] M. Woodward, B. Gouldby, Z. Kapelan, S.-T. Khu, I. Townend, Real options in flood risk management decision making, Journal of Flood Risk Management 4, (4) (2011) 339-349.

[14] A. Hooijer, F. Klijn, G. B. M. Pedroli, A. G. Van Os, Towards sustainable flood risk management in the Rhine and Meuse river basins: synopsis of the findings of IRMA-SPONGE, River Research and Applications 20 (3) (2004) 343-357.

[15] G. Di Baldassarre, A. Castellarin, A. Montanari, A. Brath, Probability-weighted hazard maps for comparing different flood risk management strategies: a case study, Natural Hazards 50 (3) (2009), 479-496.

[16] J. C. J. H. Aerts, W. Botzen, A. van der Veen, J. Krywkow, S. Werners, Dealing with uncertainty in flood management through diversification, Ecology and Society 13 (1) (2008).

[17] R. J. Dawson, T. Ball, J. Werritty, A. Werritty, J. W. Hall, N. Roche, Assessing the effectiveness of nonstructural flood management measures in the Thames Estuary under conditions of socio-economic and environmental change, Global Environmental Change 21 (2) (2011) 628-646.

[18] G. Piper, Balancing flood risk and development in the flood plain: the Lower Thames Flood Risk Management Strategy, Ecohydrology and Hydrobiology 14 (1) (2014) 33-38.

[19] K. M. De Bruijn, Resilience and flood risk management: a systems approach applied to lowland rivers, (2005).

[20] H. Zhao, S. Guo, H. Zhao, Selecting the optimal micro-grid planning program using a novel multicriteria decision-making model based on grey cumulative prospect theory, Energies 11 (7) (2018) 1840.

[21] S. Guo, H. Zhao, Fuzzy best-worst multi-criteria decision-making method and its applications, Knowledge-Based Systems 121 (2017).

[22] H. Zhang, C. Yin, X. Qi, R. Zhang, X. Kang, Cognitive best worst method for multi-attribute decisionmaking, Mathematical Problems in Engineering (2017).

[23] S. Cebi, C. Kahraman, A new weighted fuzzy information axiom method in production research, Journal of Enterprise Information Management (2019).

[24] D. E. Ighravwe, S. A. Oke, Ranking maintenance strategies for sustainable maintenance plan in manufacturing systems using fuzzy axiomatic design principle and fuzzy-TOPSIS, Journal of Manufacturing Technology Management 28 (7) (2017).

[25] M. S. Markabi, M. S. Sarbijan, A hybrid model of grey relational analysis and DEA cross-efficiency for the evaluation of decision-making units, International Journal of Economy, Management and Social Science 4 (3) (2015) 317-322.

[26] D. E. Ighravwe, S. A. Oke, A fuzzy-grey-weighted aggregate sum product assessment methodical approach for multi-criteria analysis of maintenance performance systems, International Journal of System Assurance Engineering and Management 8 (2017) 961-973.

[27] P. Rani, A. R. Mishra, A. Mardani, F. Cavallaro, M. Alrasheedi, A. Alrashidi, A novel approach to extended fuzzy TOPSIS based on new divergence measures for renewable energy sources selection, Journal of Cleaner Production 257 (2020) 120352.

[28] A. Afsordegan, M. Sánchez, N. Agell, S. Zahedi, L. V Cremades, Decision making under uncertainty using a qualitative TOPSIS method for selecting sustainable energy alternatives, International Journal of Environmental Science and Technology 13 (6) (2016) 1419-1432.

[29] D. O. Aikhuele, Reliability evaluation using MAGDM based on triangular intuitionistic attitudinal ranking and aggregating model, Journal of Modern Technology and Engineering 3(2) (2018) 165-178.

[30] NASA, "POWER Data Access Viewer," 2020. https://power.larc.nasa.gov/data-access-viewer/ (accessed Dec. 19, 2020).

[31] F. Yazdandoost, B. Bozorgy, Flood risk management strategies using multi-criteria analysis, in Proceedings of the Institution of Civil Engineers-Water Management 161(5) (2008) 261-266.

[32] S. J. Lin, I. J. Lu, C. Lewis, Grey relation performance correlations among economics, energy use and carbon dioxide emission in Taiwan, Energy Policy, 35 (3) (2007) 1948-1955. 